\title{
Further fresh and general traveling wave solutions to some fractional order nonlinear evolution equations in mathematical physics
}

Solutions to FNLEEs in mathematical physics
Tarikul Islam and Armina Akter Department of Mathematics, Hajee Mohammad Danesh Science and Technology University, Dinajpur, Bangladesh
Received 29 September 2020 Revised 13 November 2020 Accepted 19 November 2020

\begin{abstract}
Purpose - Fractional order nonlinear evolution equations (FNLEEs) pertaining to conformable fractional derivative are considered to be revealed for well-furnished analytic solutions due to their importance in the nature of real world. In this article, the autors suggest a productive technique, called the rational fractional $\left(D_{\xi}^{\alpha} G / G\right)$-expansion method, to unravel the nonlinear space-time fractional potential Kadomtsev-Petviashvili (PKP) equation, the nonlinear space-time fractional Sharma-Tasso-Olver (STO) equation and the nonlinear space-time fractional Kolmogorov-Petrovskii-Piskunov (KPP) equation. A fractional complex transformation technique is used to convert the considered equations into the fractional order ordinary differential equation. Then the method is employed to make available their solutions. The constructed solutions in terms of trigonometric function, hyperbolic function and rational function are claimed to be fresh and further general in closed form. These solutions might play important roles to depict the complex physical phenomena arise in physics, mathematical physics and engineering.

Design/methodology/approach - The rational fractional $\left(D_{\xi}^{\alpha} G / G\right)$-expansion method shows high performance and might be used as a strong tool to unravel any other FNLEEs. This method is of the form $U(\xi)=\sum_{i=0}^{n} a_{i}\left(D_{\xi}^{\alpha} G / G\right)^{i} / \sum_{i=0}^{n} b_{i}\left(D_{\xi}^{\alpha} G / G\right)^{i}$.

Findings - Achieved fresh and further abundant closed form traveling wave solutions to analyze the inner mechanisms of complex phenomenon in nature world which will bear a significant role in the of research and will be recorded in the literature.

Originality/value - The rational fractional $\left(D_{\xi}^{\alpha} G / G\right)$-expansion method shows high performance and might be used as a strong tool to unravel any other FNLEEs. This method is newly established and productive.

Keywords The rational fractional $\left(D_{\xi}^{\alpha} G / G\right)$-expansion method, Complex fractional transformation, Conformable fractional derivative, Closed form solution, Fractional order nonlinear evolution equation

Paper type Research paper
\end{abstract}

\section{Introduction}

Fractional calculus originating from some speculations of Leibniz and L'Hospital in 1695 has gradually gained profound attention of many researchers for its extensive appearance in various fields of real world. Exact traveling wave solutions to fractional order nonlinear evolution equations (FNLEEs) are of fundamental and important in applied science because of their wide use to depict the nonlinear fractional phenomena and dynamical processes of nature world. The FNLEEs and their solutions in closed form play fundamental role in describing, modeling and predicting the underlying mechanisms related to the biology, bio-genetics,

\section{JEL Classification - 34A08, 35R11}

(C) Tarikul Islam and Armina Akter. Published in Arab Journal of Mathematical Sciences. Published by Emerald Publishing Limited. This article is published under the Creative Commons Attribution (CC BY 4.0) licence. Anyone may reproduce, distribute, translate and create derivative works of this article (for both commercial and non-commercial purposes), subject to full attribution to the original publication and authors. The full terms of this licence may be seen at http://creativecommons.org/licences/by/4.0/ legalcode

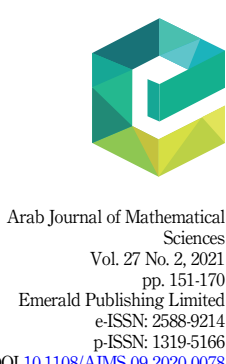

DOI 10.1108/AJMS-09-2020-0078 
AJMS

27,2

152 physics, solid state physics, condensed matter physics, plasma physics, optical fibers, meteorology, oceanic phenomena, chemistry, chemical kinematics, electromagnetic, electrical circuits, quantum mechanics, polymeric materials, neutron point kinetic model, control and vibration, image and signal processing, system identifications, the finance, acoustics and fluid dynamics [1-3]. The closed form wave solutions of these equations [4-6] are greatly helpful to realize the mechanisms of the complicated nonlinear physical phenomena as well as their further applications in practical life. Some attractive powerful approaches take into account in the recent research area related to fractional derivative associated problems [7-9]. Therefore, it has become the core aim in the research area of fractional related problems that how to develop a stable approach for investigating the solutions to FNLEEs in analytical or numerical form. Many researchers have offered different approaches to construct analytic and numerical solutions to FNLEEs as well as integer order and put them forward for searching traveling wave solutions, such as the He-Laplace method [10], the exponential decay law [11], the reproducing kernel method [12], the Jacobi elliptic function method [13], the $\left(G^{\prime} / G\right)$-expansion method and its various modifications [14-18], the exp-function method [19], the sub-equation method [20,21], the first integral method [22], the functional variable method [23], the modified trial equation method [24], the simplest equation method [25], the Lie group analysis method [26], the fractional characteristic method [27], the auxiliary equation method [28, 29], the finite element method [30], the differential transform method [31], the Adomian decomposition method [32, 33], the variational iteration method [34], the finite difference method [35], the homotopy perturbation method [36] and the He's variational principle [37], the new extended direct algebraic method [38, 39], the Jacobi elliptic function expansion method [40], the conformable double Laplace transform [41] etc. But each method does not bear high acceptance for the lacking of productivity to construct the closed form solutions to all kind of FNLEEs. That is why; it is very much indispensable to establish new techniques.

In this study, we offer a newly established technique, called the rational fractional $\left(D_{\xi}^{\alpha} G / G\right)$-expansion method [42], to investigate closed form analytic wave solutions to some FNLEEs in the sense of conformable fractional derivative [43]. This effectual and reliable productive method shows its high performance through providing abundant fresh and general solutions to the suggested equations. The obtained solutions might bring up their importance through the contribution to analyze the inner mechanisms of physical complex phenomena of real world and make an acceptable record in the literature.

\section{Preliminaries and methodology}

\subsection{Conformable fractional derivative}

A new and simple definition of derivative for fractional order introduced by Khalil et al. [43] is called conformable fractional derivative. This definition is analogous to the ordinary derivative

$$
\frac{d \psi}{d x}=\lim _{\varepsilon \rightarrow 0} \frac{\psi(x+\varepsilon)-\psi(x)}{\varepsilon},
$$

where $\psi(x):[0, \infty] \rightarrow R$ and $x>0$. According to this classical definition, $\frac{d\left(x^{n}\right)}{d x}=n x^{n-1}$. According to this perception, Khalil has introduced $\alpha$ order fractional derivative of $\psi$ as

$$
T_{\alpha} \psi(x)=\lim _{\varepsilon \rightarrow 0} \frac{\psi\left(x+\varepsilon x^{1-\alpha}\right)-\psi(x)}{\varepsilon}, 0<\alpha \leq 1,
$$

If the function $\psi$ is $\alpha$ differentiable in $(0, r)$ for $r>0$ and $\lim _{x \rightarrow 0^{+}} T_{\alpha} \psi(x)$ exists, then the conformable derivative at $x=0$ is defined as $T_{\alpha} \psi(0)=\lim _{x \rightarrow 0^{+}} T_{\alpha} \psi(x)$. The conformable integral of $\psi$ is 


$$
I_{\alpha}^{r} \psi(x)=\int_{r}^{x} \frac{\psi(t)}{t^{1-\alpha}} d t, r \geq 0,0<\alpha \leq 1 .
$$

This integral represents usual Riemann improper integral.

The conformable fractional derivative satisfies the following useful properties [43]:

If the functions $u(x)$ and $v(x)$ are $\alpha$-differentiable at any point $x>0$, for $\alpha \in(0,1]$, then

(1) $T_{\alpha}(a u+b v)=a T_{\alpha}(u)+b T_{\alpha}(v) \forall a, b \in R$.

(2) $T_{\alpha}\left(x^{n}\right)=n x^{n-\alpha} \quad \forall n \in R$.

(3) $T_{\alpha}(c)=0$, where $c$ is any constant.

(4) $T_{\alpha}(u v)=u T_{\alpha}(v)+v T_{\alpha}(u)$.

(5) $T_{\alpha}(u / v)=\frac{v T_{\alpha}(u)-u T_{\alpha}(v)}{v^{2}}$.

(6) if $u$ is differentiable, then $T_{\alpha}(u)(x)=x^{1-\alpha d u} \frac{d u}{d x}(x)$.

Many researchers used this new derivative of fractional order in physical applications due to its convenience, simplicity and usefulness [44-46].

\subsection{Methodology}

In this subsection, we discuss the main steps of the rational fractional $\left(D_{\xi}^{\alpha} G / G\right)$-expansion

method to examine exact traveling wave solutions to FNLEEs. A fractional partial differential equation in the independent variables $t, x_{1}, x_{2}, \ldots, x_{n}$ is supposed to be as follows:

$$
F\left(u_{1}, \ldots u_{k}, D_{t}^{\alpha} u_{1}, \ldots, D_{t}^{\alpha} u_{k}, D_{x_{1}}^{\beta} u_{1}, \ldots, D_{x_{1}}^{\beta} u_{k}, \ldots D_{x_{n}}^{\beta} u_{1}, \ldots D_{x_{n}}^{\beta} u_{k}, \ldots\right)=0
$$

where $0<\alpha, \beta \leq 1 ; u_{i}=u_{i}\left(t, x_{1}, x_{2}, \ldots, x_{n}\right), i=1,2,3, \ldots, k$ are unknown functions, $F$ is a polynomial in $u_{i}$ and it's various partial derivatives of fractional order. Maintain the following steps to unravel Eqn (2.2.1) by the rational fractional $\left(D_{\xi}^{\alpha} G / G\right)$-expansion technique.

Let us consider the nonlinear fractional composite transformation

$$
u_{i}=u_{i}\left(t, x_{1}, x_{2}, \ldots, x_{n}\right)=U_{i}(\xi), \xi=\xi\left(t, x_{1}, x_{2}, \ldots, x_{n}\right),
$$

which reduces Eqn (2.2.1) to the following ordinary differential equation of fractional order with respect to the variable $\xi$ :

$$
Q\left(U_{1}, \ldots, U_{k}, D_{\xi}^{\alpha} U_{1}, \ldots, D_{\xi}^{\alpha} U_{k}, D_{\xi}^{\beta} U_{1}, \ldots, D_{\xi}^{\beta} U_{k}, \ldots\right)=0 .
$$

We might take anti-derivative of Eqn (2.2.3) term by term as many times as possible and integral constant can be set to zero as soliton solutions are sought.

Step 1: Suppose the traveling wave solution of Eqn (2.2.1) can be expressed as follows:

$$
U(\xi)=\frac{\sum_{i=0}^{n} a_{i}\left(D_{\xi}^{\alpha} G / G\right)^{i}}{\sum_{i=0}^{n} b_{i}\left(D_{\xi}^{\alpha} G / G\right)^{i}},
$$

where $a_{i}^{\prime} s$ and $b_{i}, s$ are unknown constants to be determined later and $G=G(\xi)$ satisfies the following auxiliary nonlinear ordinary differential equation of fractional order:

$$
D_{\xi}^{2 \alpha} G(\xi)+\lambda D_{\xi}^{\alpha} G(\xi)+\mu G(\xi)=0,
$$

Solutions to

FNLEEs in mathematical physics 
AJMS

27,2

154

where $\lambda, \mu$ are arbitrary constants and $D_{\xi}^{\alpha} G(\xi)$ denotes the conformable fractional derivative of order $\alpha$ for $G(\xi)$ with respect to $\xi$.

The nonlinear fractional complex transformation $G(\xi)=H(\eta), \eta=\xi^{\alpha} / \Gamma(1+\alpha)$ reduces Eqn (2.2.5) into the following second order ordinary differential equation:

$$
H^{\prime \prime}(\eta)+\lambda H^{\prime}(\eta)+\mu H(\eta)=0,
$$

whose solutions are well-known. Since $D_{\xi}^{\alpha} G(\xi)=D_{\xi}^{\alpha} H(\eta)=H^{\prime}(\eta) D_{\xi}^{\alpha} \eta=H^{\prime}(\eta)$, with the aid of the solutions of Eqn (2.2.6), we can obtain the solutions of Eqn (2.2.5) as follows:

$$
\begin{gathered}
\left(D_{\xi}^{\alpha} G / G\right)=\frac{\sqrt{\lambda^{2}-4 \mu}}{2} \times \frac{C_{1} \sin h\left(\frac{\sqrt{\lambda^{2}-4 \mu} \xi^{\alpha}}{2 \Gamma(1+\alpha)}\right)+C_{2} \cosh \left(\frac{\sqrt{\lambda^{2}-4 \mu} \xi^{\alpha}}{2 \Gamma(1+\alpha)}\right)}{C_{1} \cosh \left(\frac{\sqrt{\lambda^{2}-4 \mu} \xi^{\alpha}}{2 \Gamma(1+\alpha)}\right)+C_{2} \sin h\left(\frac{\sqrt{\lambda^{2}-4 \mu} \xi^{\alpha}}{2 \Gamma(1+\alpha)}\right)}-\frac{\lambda}{2}, \quad \lambda^{2}-4 \mu>0 \\
\left(D_{\xi}^{\alpha} G / G\right)=\frac{\sqrt{4 \mu-\lambda^{2}}}{2} \times \frac{-C_{1} \sin \left(\frac{\sqrt{4 \mu-\lambda^{2}} \xi^{\alpha}}{2 \Gamma(1+\alpha)}\right)+C_{2} \cos \left(\frac{\sqrt{4 \mu-\lambda^{2}} \xi^{\alpha}}{2 \Gamma(1+\alpha)}\right)}{C_{1} \cos \left(\frac{\sqrt{4 \mu-\lambda^{2}} \xi^{\alpha}}{2 \Gamma(1+\alpha)}\right)+C_{2} \sin \left(\frac{\sqrt{4 \mu-\lambda^{2}} \xi^{\alpha}}{2 \Gamma(1+\alpha)}\right)}-\frac{\lambda}{2}, \quad \lambda^{2}-4 \mu<0 \\
\left(D_{\xi}^{\alpha} G / G\right)=\frac{C_{2} \Gamma(1+\alpha)}{C_{1} \Gamma(1+\alpha)+C_{2} \xi^{\alpha}}-\frac{\lambda}{2}, \quad \lambda^{2}-4 \mu=0
\end{gathered}
$$

where $C_{1}$ and $C_{2}$ are arbitrary constants.

Step 2: The positive constant $n$ can be determined by taking homogenous balance between the highest order linear and nonlinear terms appearing in Eqn (2.2.3).

Step 3: Substitute (2.2.4) and (2.2.5) into Eqn (2.2.3) with the value of $n$ obtained in step 2, we obtain a polynomial in $\left(D_{\xi}^{\alpha} G / G\right)$. Setting each coefficient of the resulted polynomial to zero gives a set of algebraic equations for $a_{i}^{\prime} s$ and $b_{i}, s$ by means of the symbolic computation software, such as Maple, provides the values of constants.

Step 4: Inserting the values of $a_{i}^{\prime} s$ and $b_{i}, s$ into (2.2.4) along with (2.2.7)-(2.2.9), the closed form traveling wave solutions to the nonlinear evolution Eqn (2.2.1) are obtained.

\section{Formulation of the solutions}

In this section, the exact analytic traveling wave solutions to the nonlinear space-time fractional potential Kadomtsev-Petviashvili (PKP) equation, the nonlinear space-time fractional Sharma-Tasso-Olver (STO) equation and the nonlinear space-time fractional Kolmogorov-Petrovskii-Piskunov (KPP) equation are constructed.

\subsection{The nonlinear space-time fractional PKP equation}

This well-known equation is given as

$$
\frac{1}{4} D_{x}^{4 \alpha} u+\frac{3}{2} D_{x}^{\alpha} u D_{x}^{2 \alpha} u+\frac{3}{4} D_{y}^{2 \alpha} u+D_{t}^{\alpha}\left(D_{x}^{\alpha} u\right)=0 .
$$


With the aid of the fractional compound transformation

$$
u(x, y, t)=U(\xi), \xi=x+y+c^{1 / \alpha} t
$$

Eqn (3.1.1) is turned into the following ordinary differential equations of fractional order due to the variable $\xi$ :

$$
\frac{1}{4} D_{\xi}^{4 \alpha} U+\frac{3}{2} D_{\xi}^{\alpha} U D_{\xi}^{2 \alpha} U+\frac{3}{4} D_{\xi}^{2 \alpha} U+c D_{\xi}^{2 \alpha} U=0
$$

Taking anti-derivative of (3.1.3) yields

$$
D_{\xi}^{3 \alpha} U+3\left(D_{\xi}^{\alpha} U\right)^{2}+(3+4 c) D_{\xi}^{\alpha} U=0
$$

Considering the homogenous balance to Eqn (3.1.4), the solution (2.2.4) becomes

$$
U(\xi)=\frac{a_{0}+a_{1} D_{\xi}^{\alpha} G / G}{b_{0}+b_{1} D_{\xi}^{\alpha} G / G}
$$

Eqn (3.1.4) together with (3.1.5) and (2.2.5) becomes a polynomial in $\left(D_{\xi}^{\alpha} G / G\right)$ equating whose coefficients to zero and solving provides the following outcomes:

$$
\text { set 1: } \cdot a_{0}=\frac{1}{b_{1}}\left(a_{1} b_{0}-2 b_{1}^{2} \mu+2 b_{0} b_{1} \lambda-2 b_{0}^{2}\right), c=\frac{1}{4}\left(4 \mu-\lambda^{2}-3\right),
$$

where $a_{1}, b_{0}, b_{1}, \lambda$ and $\mu$ are free parameters.

$$
\text { set 2: } a_{1}=2 b_{0}, b_{1}=0, c=\frac{1}{4}\left(4 \mu-\lambda^{2}-3\right),
$$

where $a_{0}, b_{0}, \lambda$ and $\mu$ are free parameters.

Insert the values appeared in (3.1.6) and (3.1.7) in the solution (3.1.5) provide the following expressions for exact analytic solutions:

$$
\begin{gathered}
U_{1}(\xi)=\frac{\left(a_{1} b_{0}-2 b_{1}^{2} \mu+2 b_{0} b_{1} \lambda-2 b_{0}^{2}\right)+a_{1} D_{\xi}^{\alpha} G / G}{b_{1}\left(b_{0}+b_{1} D_{\xi}^{\alpha} G / G\right)}, \\
U_{2}(\xi)=\frac{a_{0}}{b_{0}}+2 D_{\xi}^{\alpha} G / G,
\end{gathered}
$$

where $\xi=x+y+\left\{\left(4 \mu-\lambda^{2}-3\right) / 4\right\}^{1 / \alpha} t$.

The expressions (3.1.8) and (3.1.9) along with (2.2.7)-(2.2.9) make available the following closed form traveling wave solutions in terms of hyperbolic function, trigonometric function and rational function:

3.1.1 Solution 1. When $\lambda^{2}-4 \mu>0$,

$$
=\frac{\left(a_{1} b_{0}-2 b_{1}^{2} \mu+2 b_{0} b_{1} \lambda-2 b_{0}^{2}\right)+a_{1}\left(\frac{\sqrt{\lambda^{2}-4 \mu}}{2} \times \frac{C_{1} \sin h\left(\frac{\sqrt{\lambda^{2}-4 \mu} \xi^{\alpha}}{2 \Gamma(1+\alpha)}\right)+C_{2} \cos h\left(\frac{\sqrt{\lambda^{2}-4 \mu} \xi^{\alpha}}{2 \Gamma(1+\alpha)}\right)}{C_{1} \cos h\left(\frac{\sqrt{\lambda^{2}-4 \mu} \xi^{\alpha}}{2 \Gamma(1+\alpha)}\right)+C_{2} \sin h\left(\frac{\sqrt{\lambda^{2}-4 \mu} \xi^{\alpha}}{2 \Gamma(1+\alpha)}\right)}-\frac{\lambda}{2}\right)}{b_{1}\left(b_{0}+b_{1}\left(\frac{\sqrt{\lambda^{2}-4 \mu}}{2} \times \frac{C_{1} \sin h\left(\frac{\sqrt{\lambda^{2}-4 \mu} \xi^{\alpha}}{2 \Gamma(1+\alpha)}\right)+C_{2} \cos h\left(\frac{\sqrt{\lambda^{2}-4 \mu} \xi^{\alpha}}{2 \Gamma(1+\alpha)}\right)}{C_{1} \cos h\left(\frac{\sqrt{\lambda^{2}-4 \mu} \xi \xi^{\alpha}}{2 \Gamma(1+\alpha)}\right)+C_{2} \sin h\left(\frac{\sqrt{\lambda^{2}-4 \mu} \xi^{\alpha}}{2 \Gamma(1+\alpha)}\right)}-\frac{\lambda}{2}\right)\right)}
$$


AJMS

27,2

156

Choose $c_{1} \neq 0, c_{2}=0$, then (3.1.10) becomes

$$
U_{1}^{1}(\xi)=\frac{\left(a_{1} b_{0}-2 b_{1}^{2} \mu+2 b_{0} b_{1} \lambda-2 b_{0}^{2}\right)+a_{1}\left(\frac{\sqrt{\lambda^{2}-4 \mu}}{2} \times \tan h\left(\frac{\sqrt{\lambda^{2}-4 \mu} \xi^{\alpha}}{2 \Gamma(1+\alpha)}\right)-\frac{\lambda}{2}\right)}{b_{1}\left(b_{0}+b_{1}\left(\frac{\sqrt{\lambda^{2}-4 \mu}}{2} \times \tan h\left(\frac{\sqrt{\lambda^{2}-4 \mu} \xi^{\alpha}}{2 \Gamma(1+\alpha)}\right)-\frac{\lambda}{2}\right)\right)},
$$

where $\xi=x+y+\left\{\left(4 \mu-\lambda^{2}-3\right) / 4\right\}^{1 / \alpha} t$.

When $\lambda^{2}-4 \mu<0$,

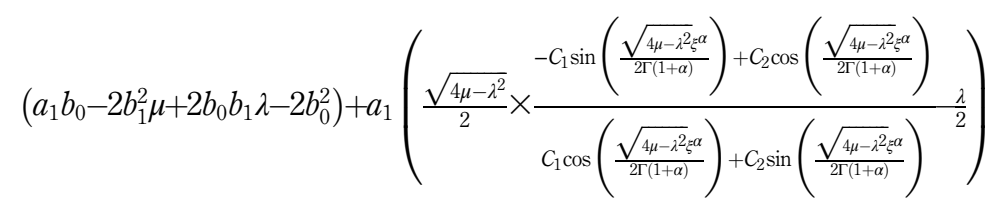

$$
\begin{aligned}
& U_{1}^{2}(\xi)=
\end{aligned}
$$

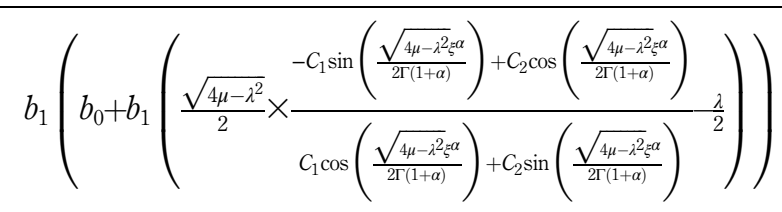

The choice of $c_{1} \neq 0, c_{2}=0$ gives way

$$
U_{1}^{2}(\xi)=\frac{\left(a_{1} b_{0}-2 b_{1}^{2} \mu+2 b_{0} b_{1} \lambda-2 b_{0}^{2}\right)-a_{1}\left(\frac{\sqrt{4 \mu-\lambda^{2}}}{2} \times \tan \left(\frac{\sqrt{4 \mu-\lambda^{2}} \xi^{\alpha}}{2 \Gamma(1+\alpha)}\right)+\frac{\lambda}{2}\right)}{b_{1}\left(b_{0}-b_{1}\left(\frac{\sqrt{4 \mu-\lambda^{2}}}{2} \times \tan \left(\frac{\sqrt{4 \mu-\lambda^{2}} \xi^{\alpha}}{2 \Gamma(1+\alpha)}\right)+\frac{\lambda}{2}\right)\right)},
$$

where $\xi=x+y+\left\{\left(4 \mu-\lambda^{2}-3\right) / 4\right\}^{1 / \alpha} t$.

When $\lambda^{2}-4 \mu=0$,

$$
U_{1}^{3}(\xi)=\frac{\left(a_{1} b_{0}-2 b_{1}^{2} \mu+2 b_{0} b_{1} \lambda-2 b_{0}^{2}\right)+a_{1}\left(\frac{C_{2} \Gamma(1+\alpha)}{C_{1} \Gamma(1+\alpha)+C_{2} \xi^{\alpha}}-\frac{\lambda}{2}\right)}{b_{1}\left(b_{0}+b_{1}\left(\frac{C_{2} \Gamma(1+\alpha)}{C_{1} \Gamma(1+\alpha)+C_{2} \xi^{\alpha}}-\frac{\lambda}{2}\right)\right)}
$$

Choosing $c_{1}=0, c_{2} \neq 0$ yields

$$
U_{1}^{3}(\xi)=\frac{\left(a_{1} b_{0}-2 b_{1}^{2} \mu+2 b_{0} b_{1} \lambda-2 b_{0}^{2}\right)-a_{1}\left(\frac{\Gamma(1+\alpha)}{\xi^{\alpha}}-\frac{\lambda}{2}\right)}{b_{1}\left(b_{0}-b_{1}\left(\frac{\Gamma(1+\alpha)}{\xi^{\alpha}}-\frac{\lambda}{2}\right)\right)},
$$

where $\xi=x+y+\{(-3) / 4\}^{1 / \alpha} t$. 
3.1.2 Solution 2. When $\lambda^{2}-4 \mu>0$,

$$
U_{2}^{1}(\xi)=\frac{a_{0}}{b_{0}}+2\left(\frac{\sqrt{\lambda^{2}-4 \mu}}{2} \times \frac{C_{1} \sinh \left(\frac{\sqrt{\lambda^{2}-4 \mu} \xi^{\alpha}}{2 \Gamma(1+\alpha)}\right)+C_{2} \cosh \left(\frac{\sqrt{\lambda^{2}-4 \mu} \xi^{\alpha}}{2 \Gamma(1+\alpha)}\right)}{C_{1} \cosh \left(\frac{\sqrt{\lambda^{2}-4 \mu} \xi^{\alpha}}{2 \Gamma(1+\alpha)}\right)+C_{2} \sinh \left(\frac{\sqrt{\lambda^{2}-4 \mu} \xi^{\alpha}}{2 \Gamma(1+\alpha)}\right)}-\frac{\lambda}{2}\right)
$$

Assigning $c_{1} \neq 0, c_{2}=0$ provides

$$
U_{2}^{1}(\xi)=\frac{a_{0}}{b_{0}}+2\left(\frac{\sqrt{\lambda^{2}-4 \mu}}{2} \times \tan h\left(\frac{\sqrt{\lambda^{2}-4 \mu} \xi^{\alpha}}{2 \Gamma(1+\alpha)}\right)-\frac{\lambda}{2}\right),
$$

where $\xi=x+y+\left\{\left(4 \mu-\lambda^{2}-3\right) / 4\right\}^{1 / \alpha} t$.

When $\lambda^{2}-4 \mu<0$,

$$
U_{2}^{2}(\xi)=\frac{a_{0}}{b_{0}}+2\left(\frac{\sqrt{4 \mu-\lambda^{2}}}{2} \times \frac{-C_{1} \sin \left(\frac{\sqrt{4 \mu-\lambda^{2}} \xi^{\alpha}}{2 \Gamma(1+\alpha)}\right)+C_{2} \cos \left(\frac{\sqrt{4 \mu-\lambda^{2}} \xi^{\alpha}}{2 \Gamma(1+\alpha)}\right)}{C_{1} \cos \left(\frac{\sqrt{4 \mu-\lambda^{2}} \xi^{\alpha}}{2 \Gamma(1+\alpha)}\right)+C_{2} \sin \left(\frac{\sqrt{4 \mu-\lambda^{2}} \xi^{\alpha}}{2 \Gamma(1+\alpha)}\right)}-\frac{\lambda}{2}\right)
$$

Conveying $c_{1} \neq 0, c_{2}=0$ offers

$$
U_{2}^{2}(\xi)=\frac{a_{0}}{b_{0}}-2\left(\frac{\sqrt{4 \mu-\lambda^{2}}}{2} \times \tan \left(\frac{\sqrt{4 \mu-\lambda^{2}} \xi^{\alpha}}{2 \Gamma(1+\alpha)}\right)+\frac{\lambda}{2}\right),
$$

where $\xi=x+y+\left\{\left(4 \mu-\lambda^{2}-3\right) / 4\right\}^{1 / \alpha} t$.

When $\lambda^{2}-4 \mu=0$,

$$
U_{2}^{3}(\xi)=\frac{a_{0}}{b_{0}}+2\left(\frac{C_{2} \Gamma(1+\alpha)}{C_{1} \Gamma(1+\alpha)+C_{2} \xi^{\alpha}}-\frac{\lambda}{2}\right),
$$

The transmission $c_{1}=0, c_{2} \neq 0$ puts forward

$$
U_{2}^{3}(\xi)=\frac{a_{0}}{b_{0}}+2 \frac{\Gamma(1+\alpha)}{\xi^{\alpha}}-\lambda,
$$

where $\xi=x+y+\{(-3) / 4\}^{1 / \alpha} t$.

\subsection{The nonlinear space-time fractional STO equation}

Consider the nonlinear space-time fractional STO equation

$$
D_{t}^{\alpha} u+3 \beta\left(D_{x}^{\alpha} u\right)^{2}+3 \beta u^{2} D_{x}^{\alpha} u+3 \beta u D_{x}^{2 \alpha} u+\beta D_{x}^{3 \alpha} u=0
$$

Using the complex fractional transformation

$$
u(x, t)=U(\xi), \xi=k^{1 / \alpha} x+c^{1 / \alpha} t,
$$

Eqn (3.2.1) reduces to the following fractional order ordinary differential equation with respect to the variable $\xi$ :

$$
c D_{\xi}^{\alpha} U+3 k^{2} \beta\left(D_{\xi}^{\alpha} U\right)^{2}+3 k \beta U^{2} D_{\xi}^{\alpha} U+3 k^{2} \beta U D_{\xi}^{2 \alpha} U+k^{3} \beta D_{\xi}^{3 \alpha} U=0,
$$


AJMS

27,2

158

Taking anti-derivative of Eqn (3.2.3) yields

$$
c U+3 k^{2} \beta U D_{\xi}^{\alpha} U+k \beta U^{3}+k^{3} \beta D_{\xi}^{2 \alpha} U=0
$$

Applying the homogeneous balance method to Eqn (3.2.4) the solution (2.2.4) takes the form (3.1.5).

Eqn (3.2.4) under the use of solution (3.1.5) and Eqn (2.2.5) creates a polynomial in $\left(D_{\xi}^{\alpha} G / G\right)$ whose coefficients assigning to zero and solving yields the outcomes:

$$
\text { Set 1: } a_{0}=\frac{b_{0}\left\{\left(b_{1} \lambda-2 b_{0}\right) k \sqrt{-k \beta c} \mp 3 b_{1} c\right\}}{ \pm\left(b_{1} \lambda-2 b_{0}\right) k^{2} \beta+3 b_{1} \sqrt{-k \beta c}}, a_{1}= \pm b_{1} \sqrt{\frac{-c}{k \beta}}
$$

where $b_{0}, b_{1}, k, c, \beta$ and $\lambda$ are all arbitrary constants.

$$
\text { Set 2: } a_{0}= \pm b_{0} \sqrt{\frac{-c}{k \beta}}, a_{1}= \pm \frac{2 b_{0} k \sqrt{-k \beta c}}{k^{2} \beta \lambda \pm 3 \sqrt{-k \beta c}}, b_{1}=\frac{2 b_{0} k^{2} \beta}{k^{2} \beta \lambda \pm 3 \sqrt{-k \beta c}},
$$

where $b_{0}, k, c, \beta$ and $\lambda$ are all unknown parameters.

Utilizing the values available in (3.2.5) and (3.2.6) in (3.1.5) provide the following expressions for analytic solutions:

$$
\begin{gathered}
U_{1}(\xi)=\frac{\frac{b_{0}\left\{\left(b_{1} \lambda-2 b_{0}\right) k \sqrt{-k \beta c}+3 b_{1} c\right\}}{ \pm\left(b_{1} \lambda-2 b_{0}\right) k^{2} \beta+3 b_{1} \sqrt{-k \beta c}} \pm b_{1} \sqrt{\frac{-c}{k \beta}}\left(D_{\xi}^{\alpha} G / G\right)}{b_{0}+b_{1}\left(D_{\xi}^{\alpha} G / G\right)}, \\
U_{2}(\xi)= \pm \frac{b_{0} \sqrt{\frac{-c}{k \beta}}+\frac{2 b_{0} k \sqrt{-k \beta c}}{k^{2} \beta \lambda \pm 3 \sqrt{-k \beta c}}\left(D_{\xi}^{\alpha} G / G\right)}{b_{0}+\frac{2 b_{0} k^{2} \beta}{k^{2} \beta \lambda \pm 3 \sqrt{-k \beta c}}\left(D_{\xi}^{\alpha} G / G\right)},
\end{gathered}
$$

where $\xi=k^{1 / \alpha} x+c^{1 / \alpha} t$.

The expressions (3.2.7) and (3.2.8) along with (2.2.7)-(2.2.9) make available the following closed form traveling wave solutions in terms of hyperbolic function, trigonometric function and rational function:

3.2.1 Solution 1. When $\lambda^{2}-4 \mu>0$,

$U_{1}^{1}(\xi)=$

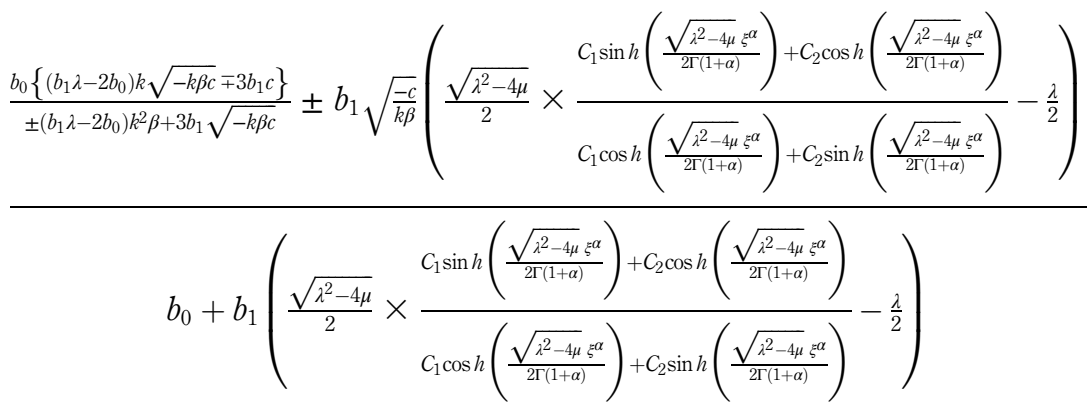


Fixing $c_{1} \neq 0, c_{2}=0$ serves

Solutions to

FNLEEs in

$$
U_{1}^{1}(\xi)=\frac{\frac{b_{0}\left\{\left(b_{1} \lambda-2 b_{0}\right) k \sqrt{-k \beta c}+3 b_{1} c\right.}{ \pm\left(b_{1} \lambda-2 b_{0}\right) k^{2} \beta+3 b_{1} \sqrt{-k \beta c}} \pm b_{1} \sqrt{\frac{-c}{k \beta}}\left(\frac{\sqrt{\lambda^{2}-4 \mu}}{2} \times \tan h\left(\frac{\sqrt{\lambda^{2}-4 \mu} \xi^{\alpha}}{2 \Gamma(1+\alpha)}\right)-\frac{\lambda}{2}\right)}{b_{0}+b_{1}\left(\frac{\sqrt{\lambda^{2}-4 \mu}}{2} \times \tan h\left(\frac{\sqrt{\lambda^{2}-4 \mu}}{2 \Gamma(1+\alpha)}\right)-\frac{\lambda}{2}\right)}
$$

mathematical

physics

where $\xi=k^{1 / \alpha} x+c^{1 / \alpha} t$.

When $\lambda^{2}-4 \mu<0$,

$$
\begin{aligned}
& \frac{b_{0}\left\{\left(b_{1} \lambda-2 b_{0}\right) k \sqrt{-k \beta c} \mp 3 b_{1} c\right\}}{ \pm\left(b_{1} \lambda-2 b_{0}\right) k^{2} \beta+3 b_{1} \sqrt{-k \beta c}} \pm b_{1} \sqrt{\frac{-c}{k \beta}}\left(\frac{\sqrt{4 \mu-\lambda^{2}}}{2} \times \frac{-C_{1} \sin \left(\frac{\sqrt{4 \mu-\lambda^{2}} \xi^{\alpha}}{2 \Gamma(1+\alpha)}\right)+C_{2} \cos \left(\frac{\sqrt{4 \mu-\lambda^{2}} \xi^{\alpha}}{2 \Gamma(1+\alpha)}\right)}{C_{1} \cos \left(\frac{\sqrt{4 \mu-\lambda^{2}} \xi^{\alpha}}{2 \Gamma(1+\alpha)}\right)+C_{2} \sin \left(\frac{\sqrt{4 \mu-\lambda^{2}} \xi^{\alpha}}{2 \Gamma(1+\alpha)}\right)}-\frac{\lambda}{2}\right) \\
& U_{1}^{2}(\xi)= \\
& b_{0}+b_{1}\left(\frac{\sqrt{4 \mu-\lambda^{2}}}{2} \times \frac{-C_{1} \sin \left(\frac{\sqrt{4 \mu-\lambda^{2}} \xi^{\alpha}}{2 \Gamma(1+\alpha)}\right)+C_{2} \cos \left(\frac{\sqrt{4 \mu-\lambda^{2}} \xi^{\alpha}}{2 \Gamma(1+\alpha)}\right)}{C_{1} \cos \left(\frac{\sqrt{4 \mu-\lambda^{2}} \xi^{\alpha}}{2 \Gamma(1+\alpha)}\right)+C_{2} \sin \left(\frac{\sqrt{4 \mu-\lambda^{2}} \xi^{\alpha}}{2 \Gamma(1+\alpha)}\right)}-\frac{\lambda}{2}\right)
\end{aligned}
$$

Setting up $c_{1} \neq 0, c_{2}=0$ provides

$$
U_{1}^{2}(\xi)=\frac{\frac{b_{0}\left\{\left(b_{1} \lambda-2 b_{0}\right) k \sqrt{-k \beta c}+3 b_{1} c\right.}{ \pm\left(b_{1} \lambda-2 b_{0}\right) k^{2} \beta+3 b_{1} \sqrt{-k \beta c}} \mp b_{1} \sqrt{\frac{-c}{k \beta}}\left(\frac{\sqrt{4 \mu-\lambda^{2}}}{2} \times \tan \left(\frac{\sqrt{4 \mu-\lambda^{2}} \xi^{\alpha}}{2 \Gamma(1+\alpha)}\right)+\frac{\lambda}{2}\right)}{b_{0}-b_{1}\left(\frac{\sqrt{4 \mu-\lambda^{2}}}{2} \times \tan \left(\frac{\sqrt{4 \mu-\lambda^{2}} \xi^{\alpha}}{2 \Gamma(1+\alpha)}\right)+\frac{\lambda}{2}\right)}
$$

where $\xi=k^{1 / \alpha} x+c^{1 / \alpha} t$.

When $\lambda^{2}-4 \mu=0$,

$$
U_{1}^{3}(\xi)=\frac{\frac{b_{0}\left\{\left(b_{1} \lambda-2 b_{0}\right) k \sqrt{-k \beta c} \mp 3 b_{1} c\right\}}{ \pm\left(b_{1} \lambda-2 b_{0}\right) k^{2} \beta+3 b_{1} \sqrt{-k \beta c}} \pm b_{1} \sqrt{\frac{-c}{k \beta}}\left(\frac{C_{2} \Gamma(1+\alpha)}{C_{1} \Gamma(1+\alpha)+C_{2} \xi^{\alpha}}-\frac{\lambda}{2}\right)}{b_{0}+b_{1}\left(\frac{C_{2} \Gamma(1+\alpha)}{C_{1} \Gamma(1+\alpha)+C_{2} \xi^{\alpha}}-\frac{\lambda}{2}\right)}
$$

Putting $c_{1}=0, c_{2} \neq 0$ gives out

$$
U_{1}^{3}(\xi)=\frac{\frac{b_{0}\left\{\left(b_{1} \lambda-2 b_{0}\right) k \sqrt{-k \beta c} \mp 3 b_{1} c\right\}}{ \pm\left(b_{1} \lambda-2 b_{0}\right) k^{2} \beta+3 b_{1} \sqrt{-k \beta c}} \mp b_{1} \sqrt{\frac{-c}{k \beta}}\left(\frac{\Gamma(1+\alpha)}{\xi^{\alpha}}-\frac{\lambda}{2}\right)}{b_{0}-b_{1}\left(\frac{\Gamma(1+\alpha)}{\xi^{\alpha}}-\frac{\lambda}{2}\right)}
$$

where $\xi=k^{1 / \alpha} x+c^{1 / \alpha} t$. 


$$
b_{0} \sqrt{\frac{-c}{k \beta}}+\frac{2 b_{0} k \sqrt{-k \beta c}}{k^{2} \beta \lambda \pm 3 \sqrt{-k \beta c}}\left(\frac{\sqrt{\lambda^{2}-4 \mu}}{2} \times \frac{C_{1} \sin h\left(\frac{\sqrt{\lambda^{2}-4 \mu \xi^{\alpha}}}{2 \Gamma(1+\alpha)}\right)+C_{2} \cos h\left(\frac{\sqrt{\lambda^{2}-4 \xi^{\alpha}}}{2 \Gamma(1+\alpha)}\right)}{C_{1} \cos h\left(\frac{\sqrt{\lambda^{2}-4 \mu} \xi^{\alpha}}{2 \Gamma(1+\alpha)}\right)+C_{2} \sin h\left(\frac{\sqrt{\lambda^{2}-4 \mu} \xi^{\alpha}}{2 \Gamma(1+\alpha)}\right)}-\frac{\lambda}{2}\right)
$$

$$
b_{0}+\frac{2 b_{0} k^{2} \beta}{k^{2} \beta \lambda \pm \sqrt{-k \beta c}}\left(\frac{\sqrt{\lambda^{2}-4 \mu}}{2} \times \frac{C_{1} \sin h\left(\frac{\sqrt{\lambda^{2}-4 \mu} \xi^{\alpha}}{2 \Gamma(1+\alpha)}\right)+C_{2} \cos h\left(\frac{\sqrt{\lambda^{2}-4 \mu} \xi^{\alpha}}{2 \Gamma(1+\alpha)}\right)}{C_{1} \cos h\left(\frac{\sqrt{\lambda^{2}-4 \mu} \xi^{\alpha}}{2 \Gamma(1+\alpha)}\right)+C_{2} \sin h\left(\frac{\sqrt{\lambda^{2}-4 \mu} \xi^{\alpha}}{2 \Gamma(1+\alpha)}\right)}-\frac{\lambda}{2}\right)
$$

Selecting $c_{1} \neq 0, c_{2}=0$ yields

$$
U_{2}^{1}(\xi)= \pm \frac{b_{0} \sqrt{\frac{-c}{k \beta}}+\frac{2 b_{0} k \sqrt{-k \beta c}}{k^{2} \beta \lambda \pm 3 \sqrt{-k \beta c}}\left(\frac{\sqrt{\lambda^{2}-4 \mu}}{2} \times \tan h\left(\frac{\sqrt{\lambda^{2}-4 \mu} \xi^{\alpha}}{2 \Gamma(1+\alpha)}\right)-\frac{\lambda}{2}\right)}{b_{0}+\frac{2 b_{0} k^{2} \beta}{k^{2} \beta \lambda \pm 3 \sqrt{-k \beta c}}\left(\frac{\sqrt{\lambda^{2}-4 \mu}}{2} \times \tan h\left(\frac{\sqrt{\lambda^{2}-4 \mu} \xi^{\alpha}}{2 \Gamma(1+\alpha)}\right)-\frac{\lambda}{2}\right)}
$$

where $\xi=k^{1 / \alpha} x+c^{1 / \alpha} t$.

When $\lambda^{2}-4 \mu<0$,

$$
U_{2}^{2}(\xi)= \pm \frac{b_{0} \sqrt{\frac{-c}{k \beta}}+\frac{2 b_{0} k \sqrt{-k \beta c}}{k^{2} \beta \lambda \pm 3 \sqrt{-k \beta c}}\left(\frac{\sqrt{4 \mu-\lambda^{2}}}{2} \times \frac{-C_{1} \sin \left(\frac{\sqrt{4 \mu-\lambda^{2}} \xi^{\alpha}}{2 \Gamma(1+\alpha)}\right)+C_{2} \cos \left(\frac{\sqrt{4 \mu-\lambda^{2}} \xi^{\alpha}}{2 \Gamma(1+\alpha)}\right)}{C_{1} \cos \left(\frac{\sqrt{4 \mu-\lambda^{2}} \xi^{\alpha}}{2 \Gamma(1+\alpha)}\right)+C_{2} \sin \left(\frac{\sqrt{4-\lambda^{2}} \xi^{\alpha}}{2 \Gamma(1+\alpha)}\right)}-\frac{\lambda}{2}\right)}{b_{0}+\frac{2 b_{0} k^{2} \beta}{k^{2} \beta \lambda \pm 3 \sqrt{-k \beta c}}\left(\frac{\sqrt{4 \mu-\lambda^{2}}}{2} \times \frac{-C_{1} \sin \left(\frac{\sqrt{4 \mu-\lambda^{2}} \xi^{\alpha}}{2 \Gamma(1+\alpha)}\right)+C_{2} \cos \left(\frac{\sqrt{4 \mu-\lambda^{2}} \xi^{\alpha}}{2 \Gamma(1+\alpha)}\right)}{C_{1} \cos \left(\frac{\sqrt{4 \mu-\lambda^{2}} \xi^{\alpha}}{2 \Gamma(1+\alpha)}\right)+C_{2} \sin \left(\frac{\sqrt{4 \mu-\lambda^{2}} \xi^{\alpha}}{2 \Gamma(1+\alpha)}\right)}-\frac{\lambda}{2}\right)}
$$

Assigning $c_{1} \neq 0, c_{2}=0$ reduces

$$
U_{2}^{2}(\xi)= \pm \frac{b_{0} \sqrt{\frac{-c}{k \beta}}-\frac{2 b_{0} k \sqrt{-k \beta c}}{k^{2} \beta \lambda \pm 3 \sqrt{-k \beta c}}\left(\frac{\sqrt{4 \mu-\lambda^{2}}}{2} \times \tan \left(\frac{\sqrt{4 \mu-\lambda^{2}} \xi^{\alpha}}{2 \Gamma(1+\alpha)}\right)+\frac{\lambda}{2}\right)}{b_{0}-\frac{2 b_{0} k^{2} \beta}{k^{2} \beta \lambda \pm 3 \sqrt{-k \beta c}}\left(\frac{\sqrt{4 \mu-\lambda^{2}}}{2} \times \tan \left(\frac{\sqrt{4 \mu-\lambda^{2}} \xi^{\alpha}}{2 \Gamma(1+\alpha)}\right)+\frac{\lambda}{2}\right)},
$$

where $\xi=k^{1 / \alpha} x+c^{1 / \alpha} t$. 
When $\lambda^{2}-4 \mu=0$,

$$
U_{2}^{3}(\xi)= \pm \frac{b_{0} \sqrt{\frac{-c}{k \beta}}+\frac{2 b_{0} k \sqrt{-k \beta c}}{k^{2} \beta \lambda \pm 3 \sqrt{-k \beta c}}\left(\frac{C_{2} \Gamma(1+\alpha)}{C_{1} \Gamma(1+\alpha)+C_{2} \xi^{\alpha}}-\frac{\lambda}{2}\right)}{b_{0}+\frac{2 b_{0} k^{2} \beta}{k^{2} \beta \lambda \pm 3 \sqrt{-k \beta c}}\left(\frac{C_{2} \Gamma(1+\alpha)}{C_{1} \Gamma(1+\alpha)+C_{2} \xi^{\alpha}}-\frac{\lambda}{2}\right)}
$$

Using $c_{1}=0, c_{2} \neq 0$, we obtain

$$
U_{2}^{3}(\xi)= \pm \frac{b_{0} \sqrt{\frac{-c}{k \beta}}+\frac{2 b_{0} k \sqrt{-k \beta c}}{k^{2} \beta \lambda \pm 3 \sqrt{-k \beta c}}\left(\frac{\Gamma(1+\alpha)}{\xi^{\alpha}}-\frac{\lambda}{2}\right)}{b_{0}+\frac{2 b_{0} k^{2} \beta}{k^{2} \beta \lambda \pm 3 \sqrt{-k \beta c}}\left(\frac{\Gamma(1+\alpha)}{\xi^{\alpha}}-\frac{\lambda}{2}\right)},
$$

where $\xi=k^{1 / \alpha} x+c^{1 / \alpha} t$.

\subsection{The nonlinear space-time fractional KPP equation}

The nonlinear space-time fractional KPP equation is

$$
D_{t}^{\alpha} u-D_{x}^{2 \alpha} u+a u+b u^{2}+c u^{3}=0
$$

The fractional complex transformation

$$
u(x, t)=U(\xi), \xi=k^{1 / \alpha} x+w^{1 / \alpha} t
$$

reduces Eqn (3.3.1) to

$$
w D_{\xi}^{\alpha} U-k^{2} D_{\xi}^{2 \alpha} U+a U+b U^{2}+c U^{3}=0
$$

Applying the homogeneous balance method to Eqn (3.3.3) the solution (2.2.4) takes the form (3.1.5).

Using Eqn (3.1.5) and Eqn (2.2.5), Eqn (3.3.3) forms a polynomial in $\left(D_{\xi}^{\alpha} G / G\right)$ whose coefficients assigning to zero and solving gives up the following outcomes:

$$
\begin{aligned}
& a_{0}=1, a_{1}=\frac{a b_{1}\left\{\left(-b \pm \sqrt{b^{2}-4 a c}\right)\left(w+\lambda k^{2}\right)-4 a b_{1} k^{2} \mu\right\}}{\left(-b \pm \sqrt{b^{2}-4 a c}\right)\left(2 a b_{1} k^{2} \mu+b w+b \lambda k^{2}\right)+2 a c\left(w+\lambda k^{2}\right)} \\
& b_{0}=\frac{-b \pm \sqrt{b^{2}-4 a c}}{2 a}
\end{aligned}
$$

where $b_{1}, k, w, \lambda$ and $\mu$ are all unknown parameters.

Inserting the values from (3.3.4) in (3.1.5) provides the following expressions for exact wave analytic solutions:

$$
U(\xi)=\frac{1+\frac{a b_{1}\left\{\left(-b \pm \sqrt{b^{2}-4 a c}\right)\left(w+\lambda k^{2}\right)-4 a b_{1} k^{2} \mu\right\}\left(D_{\xi}^{\alpha} G / G\right)}{\left(-b \pm \sqrt{b^{2}-4 a c}\right)\left(2 a b_{1} k^{2} \mu+b w+b \lambda k^{2}\right)+2 a c\left(w+\lambda k^{2}\right)}}{\frac{-b \pm \sqrt{b^{2}-4 a c}}{2 a}+b_{1}\left(D_{\xi}^{\alpha} G / G\right)}
$$

where $\xi=k^{1 / \alpha} x+w^{1 / \alpha} t$.

Eqn (3.3.5) together with (2.2.7)-(2.2.9) presents the following exact traveling wave solutions: 
AJMS

27,2

162

$$
\text { When } \lambda^{2}-4 \mu>0 \text {, }
$$

$$
\begin{aligned}
& a b_{1}\left\{\left(-b_{ \pm} \sqrt{b^{2}-4 a c}\right)\left(w+\lambda k^{2}\right)-4 a b_{1} k^{2} \mu\right\}\left(\frac{\sqrt{\lambda^{2}-4 \mu}}{2} \times \frac{C_{1} \sin h\left(\frac{\sqrt{\lambda^{2}-4 \mu} \xi^{\alpha}}{2 \Gamma(1+\alpha)}\right)+C_{2} \cos h\left(\frac{\sqrt{\lambda^{2}-4 \mu} \xi^{\alpha}}{2 \Gamma(1+\alpha)}\right)}{C_{1} \cos h\left(\frac{\sqrt{\lambda^{2}-4 \mu} \xi^{\alpha}}{2 \Gamma(1+\alpha)}\right)+C_{2} \sin h\left(\frac{\sqrt{\lambda^{2}-4 \mu} \xi^{\alpha}}{2 \Gamma(1+\alpha)}\right)}-\frac{\lambda}{2}\right)
\end{aligned}
$$

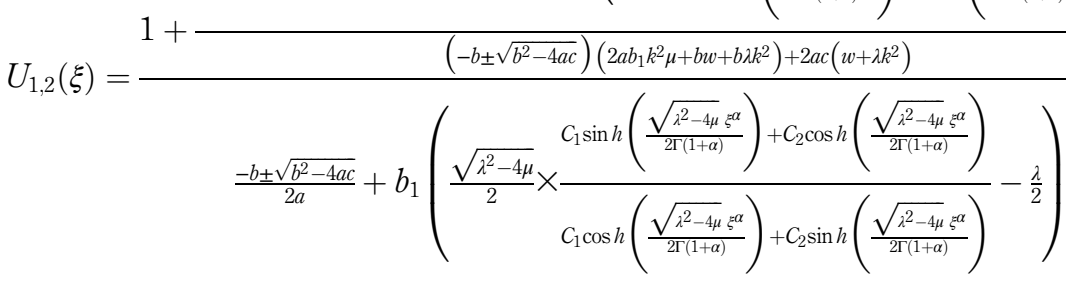

Applying $c_{1} \neq 0, c_{2}=0$ gives

$$
U_{1,2}(\xi)=\frac{1+\frac{a b_{1}\left\{\left(-b \pm \sqrt{b^{2}-4 a c}\right)\left(w+\lambda k^{2}\right)-4 a b_{1} k^{2} \mu\right\}\left(\frac{\sqrt{\lambda^{2}-4 \mu}}{2} \times \tan h\left(\frac{\sqrt{\lambda^{2}-4 \mu} \xi^{\alpha}}{2 \Gamma(1+\alpha)}\right)-\frac{\lambda}{2}\right)}{\left(-b \pm \sqrt{b^{2}-4 a c}\right)\left(2 a b_{1} k^{2} \mu+b w+b \lambda k^{2}\right)+2 a c\left(w+\lambda k^{2}\right)}}{\frac{-b \pm \sqrt{b^{2}-4 a c}}{2 a}+b_{1}\left(\frac{\sqrt{\lambda^{2}-4 \mu}}{2} \times \tan h\left(\frac{\sqrt{\lambda^{2}-4 \mu} \xi^{\alpha}}{2 \Gamma(1+\alpha)}\right)-\frac{\lambda}{2}\right)}
$$

where $\xi=k^{1 / \alpha} x+w^{1 / \alpha} t$.

When $\lambda^{2}-4 \mu<0$,

$$
U_{3,4}(\xi)=\frac{a b_{1}\left\{\left(-b \pm \sqrt{b^{2}-4 a c}\right)\left(w+\lambda k^{2}\right)-4 a b_{1} k^{2} \mu\right\}\left(\frac{\sqrt{4 \mu-\lambda^{2}}}{2} \times \frac{-C_{1} \sin \left(\frac{\sqrt{4 \mu-\lambda^{2}} \xi^{\alpha}}{2 \Gamma(1+\alpha)}\right)+C_{2} \cos \left(\frac{\sqrt{4 \mu-\lambda^{2}} \xi^{\alpha}}{2 \Gamma(1+\alpha)}\right)}{C_{1} \cos \left(\frac{\sqrt{4 \mu-\lambda^{2}} \xi^{\alpha}}{2 \Gamma(1+\alpha)}\right)+C_{2} \sin \left(\frac{\sqrt{4 \mu-\lambda^{2}} \xi^{\alpha}}{2 \Gamma(1+\alpha)}\right)}-\frac{\lambda}{2}\right)}{\frac{-b \pm \sqrt{b^{2}-4 a c}}{2 a}+b_{1}\left(\frac{\sqrt{4 \mu-\lambda^{2}}}{2} \times \frac{-C_{1} \sin \left(\frac{\sqrt{4 \mu-\lambda^{2}} \xi^{\alpha}}{2 \Gamma(1+\alpha)}\right)+C_{2} \cos \left(\frac{\sqrt{4 \mu-\lambda^{2}} \xi^{\alpha}}{2 \Gamma(1+\alpha)}\right)}{C_{1} \cos \left(\frac{\sqrt{4 \mu-\lambda^{2}}}{2 \Gamma(1+\alpha)}\right)+C_{2} \sin \left(\frac{\sqrt{4 \mu-\lambda^{2}} \xi^{\alpha}}{2 \Gamma(1+\alpha)}\right)}-\frac{\lambda}{2}\right)}
$$

Using $c_{1} \neq 0, c_{2}=0$ yields

$$
U_{3,4}(\xi)=\frac{1-\frac{a b_{1}\left\{\left(-b \pm \sqrt{b^{2}-4 a c}\right)\left(w+\lambda k^{2}\right)-4 a b_{1} k^{2} \mu\right\}\left(\frac{\sqrt{4 \mu-\lambda^{2}}}{2} \times \tan \left(\frac{\sqrt{4 \mu-\lambda^{2}} \xi^{\alpha}}{2 \Gamma(1+\alpha)}\right)+\frac{\lambda}{2}\right)}{\left(-b \pm \sqrt{b^{2}-4 a c}\right)\left(2 a b_{1} k^{2} \mu+b w+b \lambda k^{2}\right)+2 a c\left(w+\lambda k^{2}\right)}}{\frac{-b \pm \sqrt{b^{2}-4 a c}}{2 a}-b_{1}\left(\frac{\sqrt{4 \mu-\lambda^{2}}}{2} \times \tan \left(\frac{\sqrt{4 \mu-\lambda^{2}} \xi^{\alpha}}{2 \Gamma(1+\alpha)}\right)+\frac{\lambda}{2}\right)},
$$

where $\xi=k^{1 / \alpha} x+w^{1 / \alpha} t$. 
When $\lambda^{2}-4 \mu=0$,

$$
U_{5,6}(\xi)=\frac{1+\frac{a b_{1}\left\{\left(-b \pm \sqrt{b^{2}-4 a c}\right)\left(w+\lambda k^{2}\right)-4 a b_{1} k^{2} \mu\right\}\left(\frac{C_{2} \Gamma(1+\alpha)}{C_{1} \Gamma(1+\alpha)+C_{2} \xi^{-}}-\frac{\lambda}{2}\right)}{\left(-b \pm \sqrt{b^{2}-4 a c}\right)\left(2 a b_{1} k^{2} \mu+b w+b \lambda k^{2}\right)+2 a c\left(w+\lambda k^{2}\right)}}{\frac{-b \pm \sqrt{b^{2}-4 a c}}{2 a}+b_{1}\left(\frac{C_{2} \Gamma(1+\alpha)}{C_{1} \Gamma(1+\alpha)+C_{2} \xi^{\alpha}}-\frac{\lambda}{2}\right)}
$$

Solutions to

FNLEEs in mathematical physics

Fixing $c_{1}=0, c_{2} \neq 0$ gives way

$$
U_{5,6}(\xi)=\frac{1+\frac{a b_{1}\left\{\left(-b \pm \sqrt{b^{2}-4 a c}\right)\left(w+\lambda k^{2}\right)-4 a b_{1} k^{2} \mu\right\}\left(\frac{\Gamma(1+\alpha)}{\xi^{\alpha}}-\frac{\lambda}{2}\right)}{\left(-b \pm \sqrt{b^{2}-4 a c}\right)\left(2 a b_{1} k^{2} \mu+b w+b \lambda k^{2}\right)+2 a c\left(w+\lambda k^{2}\right)}}{\frac{-b \pm \sqrt{b^{2}-4 a c}}{2 a}+b_{1}\left(\frac{\Gamma(1+\alpha)}{\xi^{\alpha}}-\frac{\lambda}{2}\right)},
$$

where $\xi=k^{1 / \alpha} x+w^{1 / \alpha} t$.

\section{Graphical representations}

Some of the furnished solutions in this paper are depicted graphically for their physical appearance which stands for different shapes of soliton, like, kink-type soliton, singular kinktype soliton, periodic soliton, singular periodic soliton etc. The solution (3.1.11) represents the shape of kink-type soliton for $\lambda=4, \mu=b_{1}=3, b_{0}=2.9, a_{1}=1.9, \alpha=1$ and $y=0$ within $-10 \leq x, t \leq 10$ shown in Figure 1. Eqn (3.1.13) stands for the singular periodic soliton for $\lambda=2, \alpha=\mu=1, b_{0}=b_{1}=2, a_{1}=5$ and $x=0$ within $-10 \leq y, t \leq 10$, Eqn (3.1.15) takes the form of singular kink shape soliton for $\lambda=2, \mu=1, b_{0}=4, b_{1}=3, a_{1}=1.5, \alpha=0.5$ and $y=0$ in the range $-10 \leq x, t \leq 10$ exposed in Figure 2. Eqn (3.1.17) represents kink-type soliton for $\lambda=4, \mu=3, \alpha=b_{0}=1$ and $a_{0}=0.5$ within $-10 \leq x, t \leq 10$, Eqn (3.1.19) gives

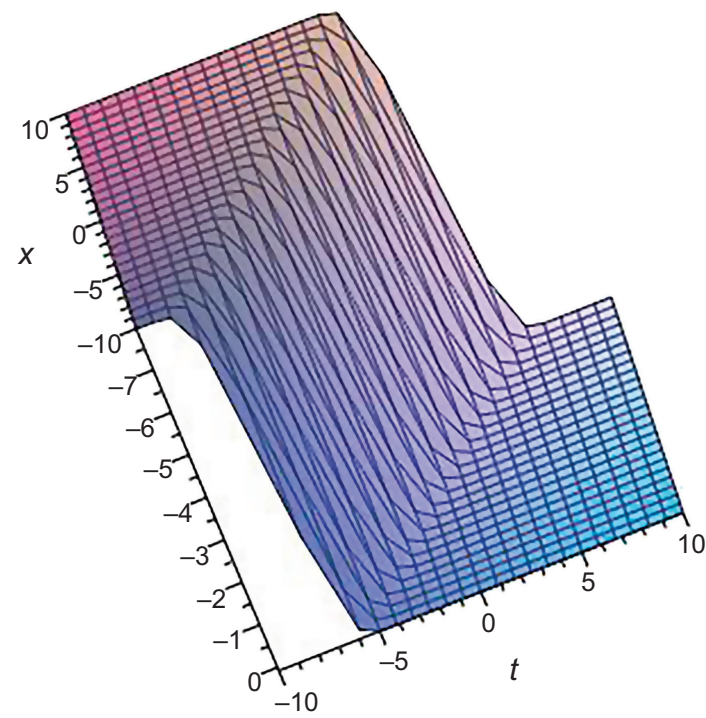

Figure 1.

Kink-type soliton of solution (3.1.11) for $\lambda=4, \mu=b_{1}=3$, $b_{0}=2.9, a_{1}=1.9$ $\alpha=1$ and $y=0$ in $-10 \leq x, t \leq 10$ 
AJMS
27,2

164

Figure 2.

Shape of solution (3.1.15) for $\lambda=2$, $\mu=1, b_{0}=4$, $b_{1}=3, a_{1}=1.5$, $\alpha=0.5$ and $y=0$ in the

range $-10 \leq x, t \leq 10$

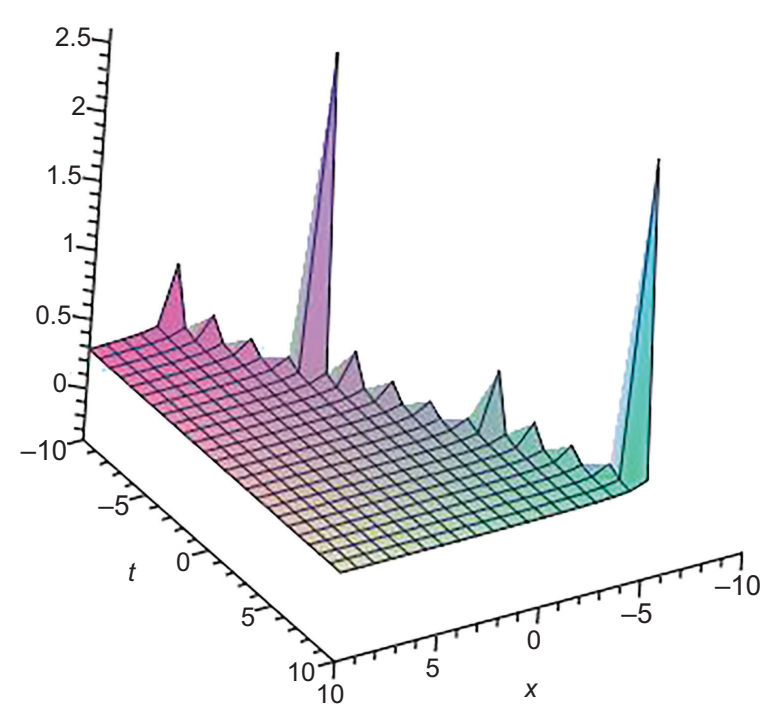

the shape of periodic soliton for $\lambda=3, \mu=2.5, b_{0}=0.5, a_{0}=1, \alpha=1$ and $y=0$ in the interval $-10 \leq x, t \leq 10$ given away in Figure 3. Eqn (3.1.21) stands for the singular periodic soliton for $\alpha=\lambda=a_{0}=1, b_{0}=0.5$ and $y=0$ within the range $-10 \leq x, t \leq 10$. The solution (3.2.10) represents the kink-type soliton for $\lambda=4, \mu=b_{1}=3, \beta=b_{0}=2, \alpha=c=1$ and $k=-1$ within $-10 \leq x, t \leq 10$. Eqn (3.2.12) stands for periodic soliton with $\lambda=2, \mu=5$, $b_{0}=2, b_{1}=3, \alpha=\beta=1, k=-1$ and $c=2$ in the interval $-10 \leq x, t \leq 10$ shown in Figure 4. Eqn (3.2.14) presents singular kink soliton for $\lambda=2, \mu=5, b_{0}=0.2$, $b_{1}=0.3, \alpha=k=c=1$ and $\beta=-2$ within the range $-10 \leq x, t \leq 10$ revealed in Figure 5 .

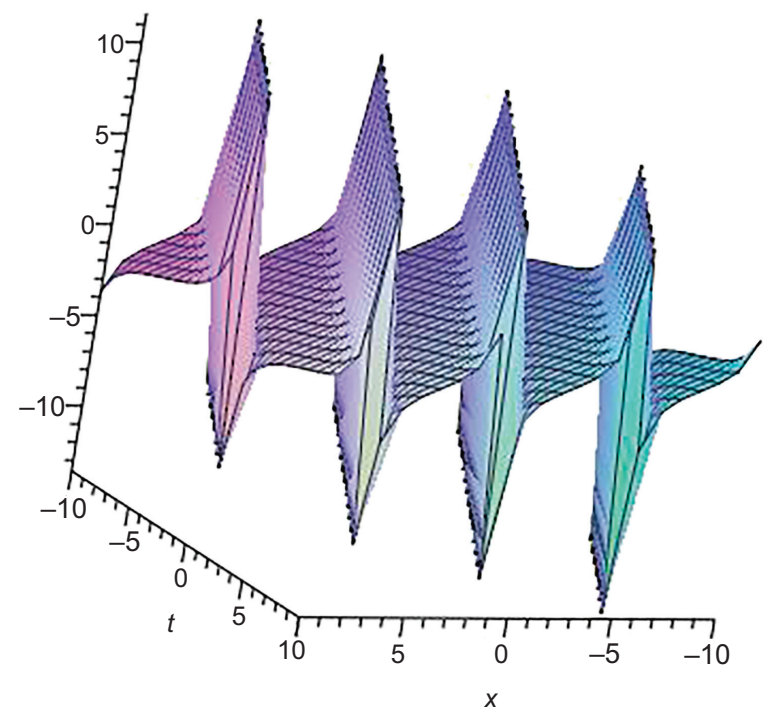

Figure 3.

Periodic plot of solution (3.1.19)

for $\lambda=3$,

$\mu=2.5, b_{0}=0.5$

$a_{0}=1, \alpha=1$ and $y=0$

within $-10 \leq x, t \leq 10$ 
Eqn (3.2.16) takes the form of kink-type soliton for $\lambda=4, \mu=3, \alpha=k=1, c=2$, $b_{0}=0.5, b_{1}=1.5$ and $\beta=-1$ with $-10 \leq x, t \leq 10$. Eqn (3.2.18) gives the shape of periodic soliton for $\lambda=b_{0}=2, \mu=5, \alpha=k=c=1, b_{1}=3$ and $\beta=-2$ in the interval $-10 \leq x, t \leq 10$. Eqn (3.2.20) represents singular kink-type soliton for $\lambda=2, \mu=k=c=1$, $b_{0}=0.2, b_{1}=0.3, \alpha=0.5$ and $\beta=-2$ within $-10 \leq x, t \leq 10$ shown in Figure 6 . The solution (3.3.7) represents the kink-type soliton for $\lambda=4, \mu=3, a_{1}=b_{0}=0.5$, $b_{1}=1.5, \alpha=k=w=p=r=1$ and $q=2$ in the range $-10 \leq x, t \leq 10$ made known in Figure 7. Eqn (3.3.9) stands for periodic soliton for $\lambda=2, \mu=5, b_{0}=0.2, \alpha=k=w$ $=p=r=1, a_{1}=0.5, b_{1}=0.2$ and $q=2.5$ within the interval $-10 \leq x, t \leq 10$ given away
Solutions to FNLEEs in mathematical physics

165

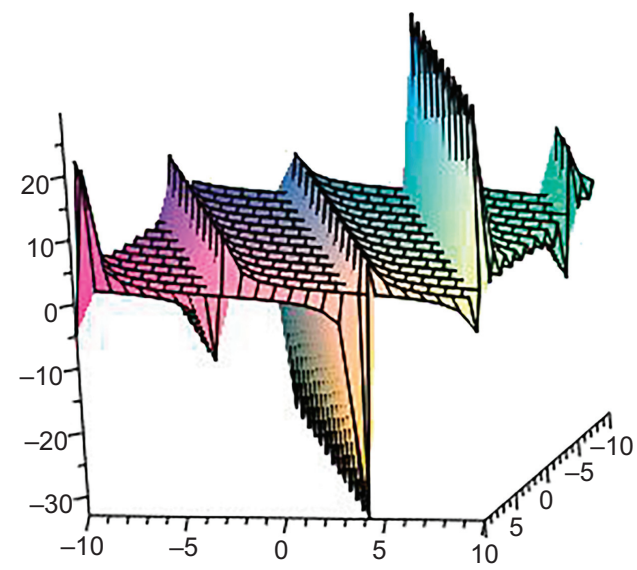

Figure 4.

Physical appearance of solution (3.2.12) for

$\lambda=2, \mu=5$,

$b_{0}=2, b_{1}=3$,

$\alpha=\beta=1, k=-1$

and $c=2$

in $-10 \leq x, t \leq 10$

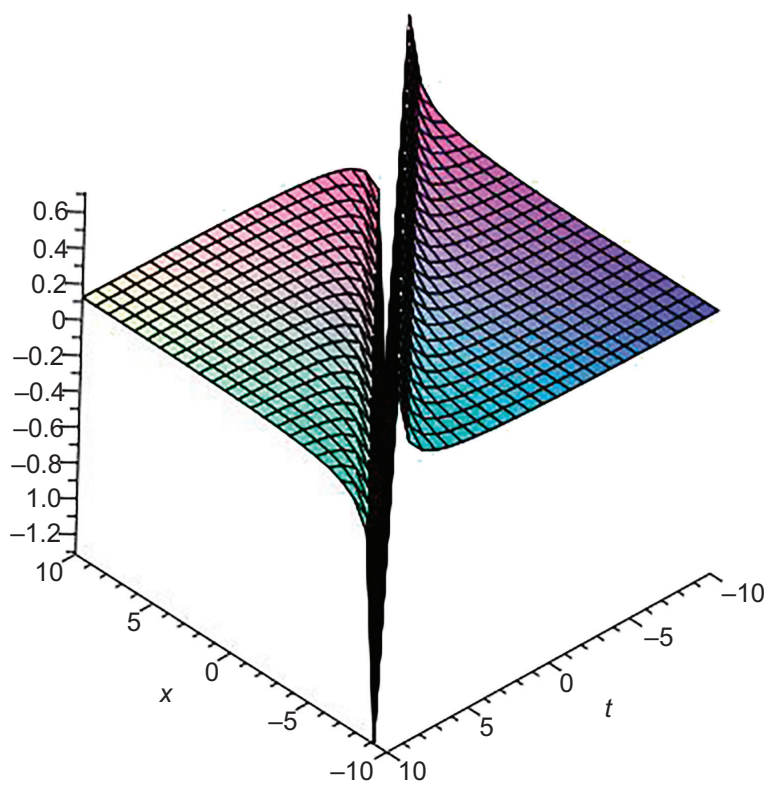

Figure 5.

Singular kink-type soliton of solution

(3.2.14) for

$\lambda=2, \mu=5$,

$b_{0}=0.2, b_{1}=0.3$,

$\alpha=k=c=1$ and

$\beta=-2$ in the

range $-10 \leq x, t \leq 10$ 


\section{AJMS \\ 27,2}

166

Figure 6.

Plot of solution (3.2.20)

for $\lambda=2$,

$\mu=k=c=1$,

$b_{0}=0.2, b_{1}=0.3$,

$\alpha=0.5$, and

$\beta=-2$ within

$-10 \leq x, t \leq 10$

\section{Figure 7.}

Physical appearance of solution (3.3.7) for $\lambda=4, \mu=3$,

$a_{1}=b_{0}=0.5$,

$b_{1}=1.5, \alpha=k=$

$w=p=r=1$ and

$q=2$ in the

interval

$-10 \leq x, t \leq 10$
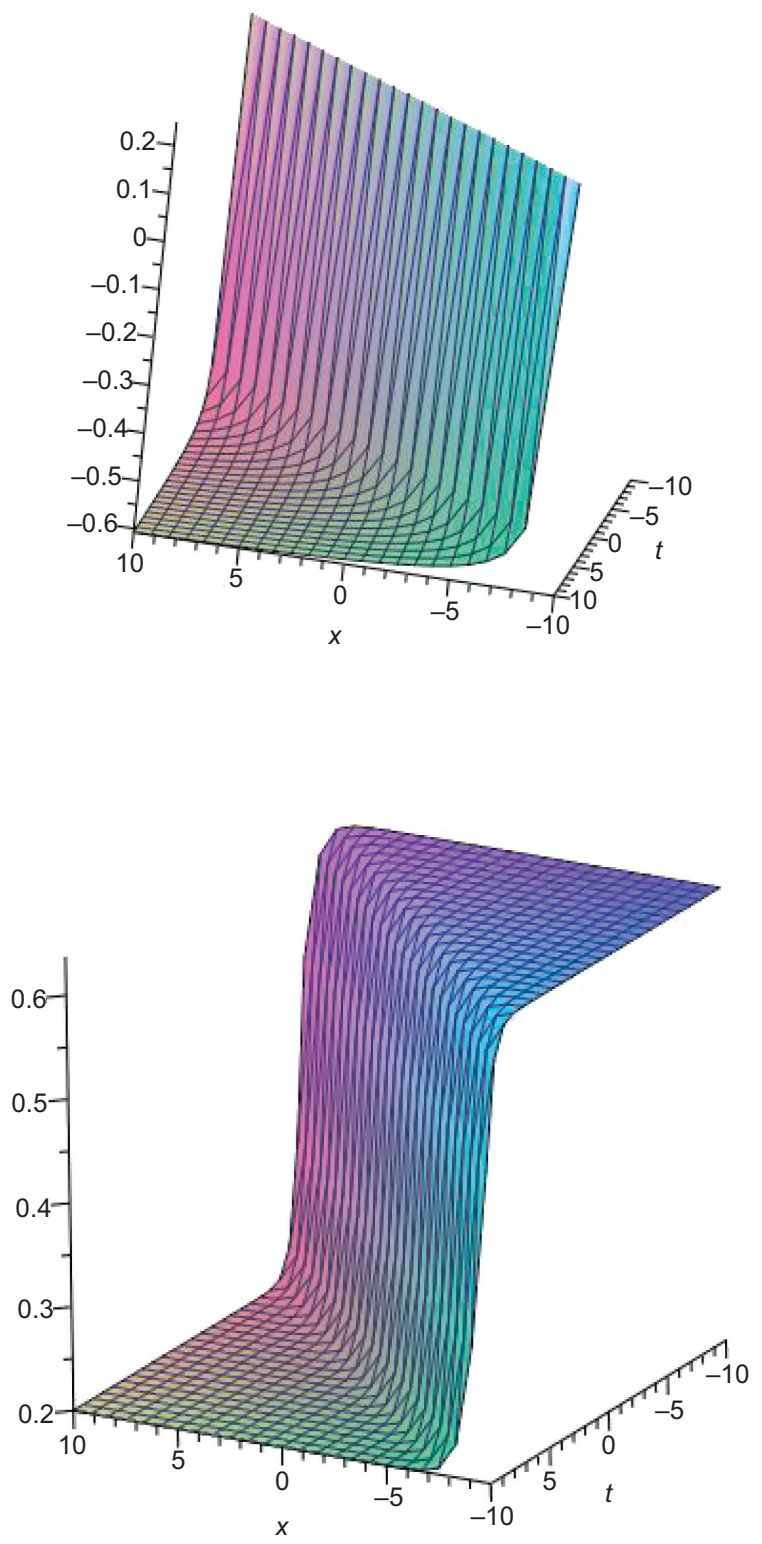

in Figure 8. Eqn (3.3.11) takes the form of singular kink-type soliton for $\lambda=2, \alpha=\mu=w$ $=k=r=1, q=2, b_{0}=0.4, b_{1}=0.2$ and $p=0.5$ in the range $-10 \leq x, t \leq 10$ exposed in Figure 9.

The physical appearance of solutions to FNLEEs bears great importance to depict different phenomena arisen in various fields of nature in real world. This paper consists of some fresh and general solutions among which few are graphically brought up. 


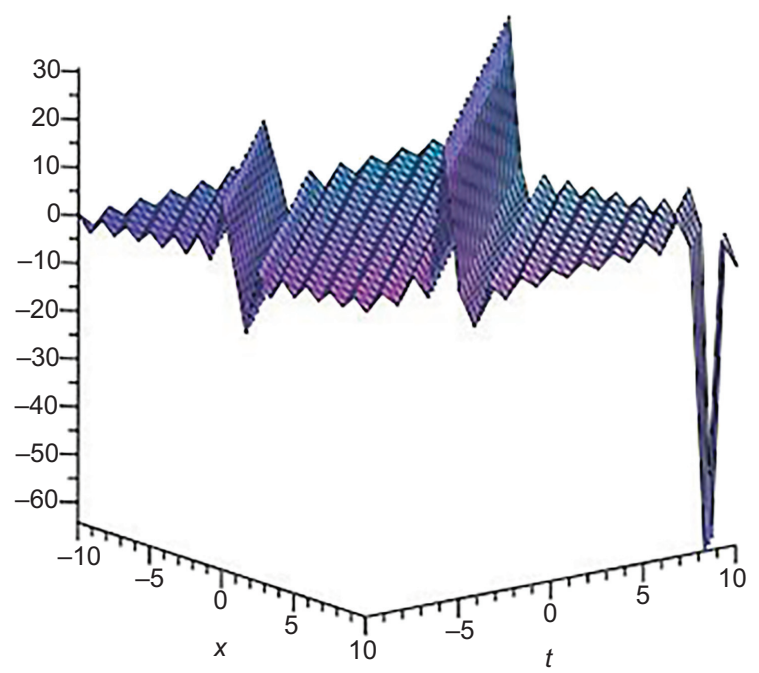

Solutions to

FNLEEs in mathematical physics

Figure 8.

Periodic shape of solution (3.3.9) for

$\lambda=2, \mu=5$,

$b_{0}=0.2, \alpha=k=w$ $=p=r=1$,

$a_{1}=0.5, \quad b_{1}=0.2$ and $q=2.5$ within the range $-10 \leq x, t \leq 10$

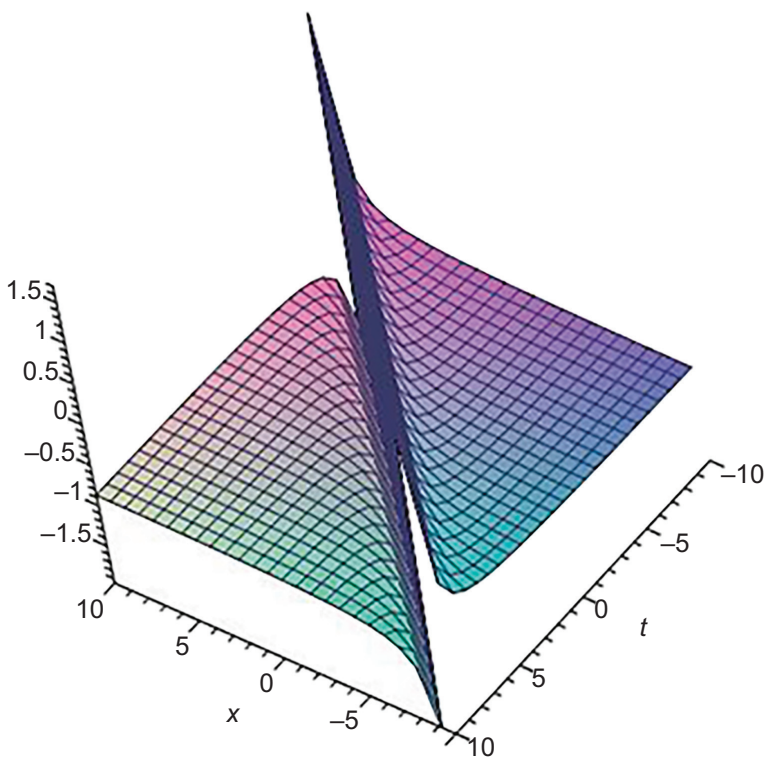

Figure 9.

Plot of solution (3.3.11) for $\lambda=2, \alpha=\mu=$ $w=k=r=1, q=2$, $b_{0}=0.4, b_{1}=0.2$ and $p=0.5$ within the interval $-10 \leq x, t \leq 10$

\section{Conclusion}

The core aim of this study is to make available further general and fresh closed form analytic wave solutions to the nonlinear space-time fractional PKP equation, the nonlinear space-time fractional STO equation and the nonlinear space-time fractional KPP equation through the suggested rational fractional $\left(D_{\xi}^{\alpha} G / G\right)$-expansion method. The offered method has successfully presented attractive solutions to the considered equations and shown its high performance. So far we know the achieved solutions are not available in the literature and 
AJMS

27,2

168 might create a milestone in research area to analyze the physical structure and behavior of the real life events that correspond to the fractional related models. Therefore, it may be claimed that the rational fractional $\left(D_{\xi}^{\alpha} G / G\right)$-expansion method in deriving the closed form analytical solutions is simple, straightforward and productive. This method might be taken into account for further implementation to investigate any FNLEEs arising in various fields of applied mathematics and mathematical physics. The obtained solutions in terms of trigonometric function, hyperbolic function and rational function containing many free parameters are claimed to be fresh and further general which will take place in the literature.

\section{References}

1. Oldham KB, Spanier J. The fractional calculus. NewYork, NY: Academic Press; 1974.

2. Samko G, Kilbas AA, Marichev OI. Fractional integrals and derivatives, Theor Appl. Yverdon: Gordan and Breach; 1993.

3. Podlubny I. Fractional differential equations, vol. 198 of mathematics in science and engineering. San Diego, CA: Academic Press; 1999.

4. Baleanu D, Diethelm K, Scalas E, Trujillo JJ. Fractional calculus: models and numerical methods, vol. 3 of series on complexity, nonlinearity and chaos. Boston, Mass: World Scientific Publishing; 2012.

5. Yang XJ. Advanced local fractional calculus and its applications. New York, NY: World Science Publisher; 2012.

6. Mainardi F. Fractional calculus and waves in linear viscoelasticity: an introduction to mathematical models. London: Imperial College Press; 2010.

7. He JH, Ji FY. Two-scale mathematics and fractional calculus for thermodynamics. Therm Sci. 2019; 23: 2131-2133.

8. He JH, Elagan SK, Li Z. explanation of the fractional complex transform and derivative chain rule for fractional calculus. Phys Lett A. 2012; 376: 257-259.

9. He JH. Jin X, A short review on analytical methods for the capillary oscillator in a nanoscale deformable tube, Math Meth Appl Sci., 2020; 43: doi: 10.1002/mma.6321.

10. Li F, Nadeem M. He-Laplace method for nonlinear vibration in shallow water waves. J Low Freq Noise Vib Act Cont. 2019; 38: 1305-1313.

11. Atangana A, Aguilar JFG. Numerical approximation of Riemann-Liouville definition of fractional derivative: from Riemann-Liouville to Atangana-Baleanu. Numer Meth Partial Diff Eq. 2017; 34: doi: 10.1002/num.22195.

12. Akgul A, Baleanu D, Inc M, Tchier F. On the solutions of electrohydrodynamic flow with fractional differential equations by reproducing kernel method. Open Phys. 2017; 128: 218-223.

13. Aslan EC, Inc M. Soliton solutions of NLSE with quadratic-cubic nonlinearity and stability analysis. Waves Rand Comp Media. 2017; 27: 594-601.

14. Islam MT, Akbar MA, Azad MAK. Traveling wave solutions to some nonlinear fractional partial differential equations through the rational -expansion method. J Ocean Engr Sci. 2018; 3: $76-81$.

15. Inan IE, Ugurlu Y, Inc M. New applications of the -expansion method. Acta Phys Pol A. 2015; 128: 245-251.

16. Islam MT, Akbar MA, Azad MAK. Traveling wave solutions in closed form for some nonlinear fractional evolution equations related to conformable fractional derivative. AIMS Mathematics. 2018; 3(4): 625-646.

17. Baleanu D, Ugurlu Y, Inc M, Kilic B. Improved -expansion method for the time fractional Biological population model and Cahn-Hilliard equation, J Comput Nonlin Dynam. 2015; 10: 051016 . 
18. Islam MT, Akbar MA, Azad, MAK. A Rational -expansion method and its application to the modified KdV-Burgers equation and the (2+1)-dimensional Boussinesq equation. Nonlinear Stud. 2015; 6: 1-11.

19. Guner O, Bekir A, Bilgil, H. A note on Exp-function method combined with complex transform method applied to fractional differential equations. Adv Nonlinear Anal. 2015; 4: 201-208.

Solutions to

FNLEEs in mathematical physics

21. Kurt A. New analytical and numerical results for fractional Bogoyavlensky-Konopelchenko equation arising in fluid dynamics. Appl Math J Chinese Univ. 2020; 35: 101-112.

22. Martinez HY, Aguilar JFG. Atangana, A. First integral method for nonlinear differential equations with conformable derivative. Math Model Nat Phenom. 2018; 13.

23. Inc, M, Inan IE, Ugurlu Y. New applications of the functional variable method. Optik. 2017; 136: 374-381.

24. Bulut H, Baskonus HM, Pandir Y. The modified trial equation method for fractional wave equation and time fractional generalized Burgers equation. Abstr Appl Anal. 2013; 2013: 636802.

25. Taghizadeh N, Mirzazadeh M, Rahimian M, Akbari M. 2013. Application of the simplest equation method to some time fractional partial differential equations. Ain Shams Eng J.; 4: 897-902.

26. Chen C, Jiang YL. Lie group analysis method for two classes of fractional partial differential equations. Commun. Nonlinear Sci Numer Simul. 2015; 26: 24-35.

27. Wu GC. A fractional characteristic method for solving fractional partial differential equations. Appl Math Lett. 2011; 24: 1046-1050.

28. Seadawy AR. Travelling-wave solutions of a weakly nonlinear two-dimensional higher-order Kadomtsev-Petviashvili dynamical equation for dispersive shallow-water waves. Eur Phys J Plus. 2017; 2017: 29, 132.

29. Akbulut A, Kaplan M, Bekir A. Auxiliary equation method for fractional differential equations with modified Riemann-Liouville derivative. Int J Nonlinear Sci Numer Simul. 2016; 17: doi: 10. 1515/ijnsns-2016-0023.

30. Deng W. Finite element method for the space and time fractional Fokker-Planck equation. SIAM J Numer Anal. 2008; 47: 204-226.

31. Momani S, Odibat Z, Erturk VS. Generalized differential transform method for solving a spaceand time-fractional diffusion-wave equation. Phys Lett A. 2007; 370: 379-387.

32. Hu Y., Luo Y., Lu Z. Analytical solution of the linear fractional differential equation by Adomian decomposition method. J Comput Appl Math. 2008; 215: 220-229.

33. El-Sayed AMA, Behiry SH, Raslan WE. Adomian's decomposition method for solving an intermediate fractional advection-dispersion equation. Comput Math Appl. 2010; 59: 1759-1765.

34. Inc M. The approximate and exact solutions of the space- and time-fractional Burgers equations with initial conditions by variational iteration method. J Math Anal Appl. 2008; 345: 476-484.

35. Gao GH., Sun ZZ, Zhang YN. A finite difference scheme for fractional sub-diffusion equations on an unbounded domain using artificial boundary conditions, J Comput Phys. 2012; 231: 2865-2879.

36. Gepreel KA. The homotopy perturbation method applied to nonlinear fractional KadomtsevPetviashvili-Piskkunov equations, Appl Math Lett. 2011; 24: 1458-1434.

37. Inc M. Some special structures for the generalized nonlinear Schrodinger equation with nonlinear dispersion. Waves Rand Comp Media. 2013; 23: 77-88.

38. Tozar A, Kurt A, Tasbozan O. New wave solutions of an integrable dispersive wave equation with a fractional time derivative arising in ocean engineering models. Kuwait J Sci. 2020; 47: 22-33.

39. Kurt A, Tozar A, Tasbozan O. Applying the new extended direct algebraic method to solve the equation of obliquely interacting waves in shallow water. J Ocean Univ China. 2020; 19: 772-780. 
AJMS

27,2

40. Tasbozan O. New analytical solutions for time fractional Benjamin-Ono Equation arising internal waves in deep water. China Ocean Eng. 2019; 33: 593-600.

41. Ozkan O, Kurt A. Conformable fractional double laplace transform and its applications to fractional partial integro-differential equations. J Frac Cal Appl. 2020; 11: 70-81.

42. Islam MT, Akbar MA, Azad MAK. Closed-form travelling wave solutions to the nonlinear spacetime fractional coupled Burgers' equation. Arab J Basic Appl. Sci. 2019; 26: 1-11.

43. Khalil R, Al Horani M, Yousef A, Sababheh MAM. A new definition of fractional derivative. J Comput Appl Math. 2014; 264: 65-70.

44. Atangana A, Baleanu D, Alsaedi A. New properties of conformable derivative. Open Math. 2015; 13(1): 889-898.

45. Eslami M, Rezazadeh $\mathrm{H}$. The first integral method for Wu-Zhang system with conformable timefractional derivative, Calcolo. 2016; 53: 475-85.

46. Cenesiz Y, Kurt A. The new solution of time fractional wave equation with conformable fractional derivative definition. J New Theory. 2015; 7: 79-85.

\section{Corresponding author}

Tarikul Islam can be contacted at: tarikul_hstu@yahoo.com

For instructions on how to order reprints of this article, please visit our website:

www.emeraldgrouppublishing.com/licensing/reprints.htm

Or contact us for further details: permissions@emeraldinsight.com 